\title{
Non-invasive brain stimulation in chronic orofacial pain: a systematic review
}

This article was published in the following Dove Press journal:

Journal of Pain Research

\author{
Alberto Herrero Babiloni ${ }^{1}$ \\ Samuel Guay' \\ Donald R Nixdorf 2,3 \\ Louis de Beaumont ${ }^{\prime}$ \\ Gilles Lavigne' \\ 'Research Center, Hôpital du Sacré- \\ Coeur de Montréal, CIUSSS du \\ Nord-de-l'île-de-Montréal, Université \\ De Montreal, Montreal, QC, Canada; \\ 2Division of TMD \& Orofacial Pain, \\ School of Dentistry, University of \\ Minnesota, Minneapolis, MN, USA; \\ ${ }^{3}$ Department of Neurology, Medical \\ School, University of Minnesota, \\ Minneapolis, MN, USA
}

Correspondence: Alberto Herrero Babiloni

Research Center, Hôpital du SacréCoeur de Montréal, CIUSSS du Nordde-l'Île-de-Montréal, Université De Montreal, 5400 Boul Gouin O, Montréal, QC H4J IC5, Canada

$\mathrm{Tel}+\mathrm{I} 5$ I4 3382222

Fax + I $514238253 \mid$

Email herre220@umn.edu
Background: Transcranial magnetic stimulation (TMS) and transcranial direct current stimulation (tDCS) are non-invasive brain stimulation techniques that are being explored as therapeutic alternatives for the management of various chronic pain conditions.

Objective: The primary objective of this systematic review is to assess the efficacy of TMS and tDCS in reducing clinical pain intensity in chronic orofacial pain (OFP) disorders. The secondary objectives are to describe adverse effects, duration of relief, and TMS/tDCS methodologies used in chronic OFP disorders.

Methods: A search was performed in MEDLINE, Embase, Web of Science, Scopus, and Google Scholar. Inclusion criteria were 1) population: adults diagnosed with chronic OFP including neuropathic and non-neuropathic disorders; 2) intervention: active TMS or tDCS stimulation regardless of the used protocol; 3) comparison: sham TMS or tDCS stimulation; and 4) outcome: primary outcome was patient reported pain intensity. Secondary outcomes were duration of pain relief, adverse effects, and methodological parameters. Risk of bias and quality of study reporting were also assessed.

Results: A total of 556 individual citations were identified by the search strategy, with 14 articles meeting selection criteria $(\mathrm{TMS}=11$; $\mathrm{tDCS}=3$ ). Data were obtained for a total of 228 patients. Included OFP disorders were trigeminal neuralgia, trigeminal neuropathy, burning mouth syndrome, atypical facial pain, and temporomandibular disorders. Significant pain reductions were obtained in both techniques. More number of sessions yielded to more durable effects. Overall, high risk of bias and poor study quality were found.

Conclusion: TMS and tDCS appear to be safe and promising alternatives to reduce pain intensity in different chronic OFP disorders. Additional research effort is needed to reduce bias, improve quality, and characterize optimal brain stimulation parameters to promote their efficacy. Keywords: transcranial magnetic stimulation, transcranial direct current stimulation, cortex, treatment, facial pain

\section{Introduction}

Chronic orofacial pain (OFP) is an umbrella term to describe different non-remittent painful disorders located in the face and/or inside the mouth. ${ }^{1}$ OFP has been estimated to affect approximately 39 million adults, or $22 \%$ of the population, in the USA. ${ }^{2}$ When OFP becomes chronic ( $\geq 6$ months), it is frequently associated with psychological distress, disability, and poor quality of life, ${ }^{3,4}$ making its treatment difficult and often refractory to conventional therapies and resulting in a high economic burden. ${ }^{5}$

Transcranial magnetic stimulation (TMS) is a non-invasive brain stimulation technique that uses changes in magnetic fields to increase or decrease neuronal activity. ${ }^{6}$ Its 
application is delivered through a magnetic coil placed over the head that transforms electrical current into a magnetic field, which can be focalized to different areas of the brain. In broad terms, different effects can be obtained depending on what area is stimulated and what frequency is used. ${ }^{7}$ For example, low frequencies $(\leq 1 \mathrm{~Hz})$ can induce neuronal inhibitory function, whereas high frequencies $(\geq 5 \mathrm{~Hz})$ are typically associated with increased cortical excitability. Repeated stimulation to the left prefrontal cortex is frequently associated with antidepressant and mood stabilizer effects, while repeated stimulation of the primary motor cortex (M1), which is usually applied to the contralateral side of the painful area, can produce analgesic effects. ${ }^{8}$ It is thought that the analgesic effects of M1 stimulation may be mediated by the activation of horizontal fibers in the superficial layers of the precentral gyrus. ${ }^{9}$ It has been demonstrated that when TMS current is propagated throughout the brain from anterior to posterior, TMS can generate late I-waves, and this orientation has been associated with pain relief., ${ }^{9,10}$ Another TMS modality is theta burst stimulation (TBS), which delivers a significantly higher number of stimulation bursts within a shorter period of time ( $<2 \mathrm{~min}$ ) when compared to conventional repetitive TMS (rTMS) protocols. This stimulation may be continuous (cTBS), leading toward a cortical inhibitory effect, or intermittent (iTBS), which has excitatory effects. ${ }^{1-13}$ Human response to TBS is influenced by the intra- and interindividual variability of cortical excitability. It is likely that the same pattern of stimulation can trigger different effects in different subjects, probably also depending on the physiological resting state of cortical neurons at the time of stimulation. The neurobiological mechanisms of TBS may involve long-term potentiation (LTP) and depression (LTD)-like processes, as well as inhibitory mechanisms modulated by GABAergic activity. ${ }^{11,13}$ Therefore, although their mechanisms are not fully understood, different rTMS techniques are emerging as alternative treatment options to manage chronic pain disorders, including neuropathic pain, fibromyalgia, and headache disorders, ${ }^{13}$ as well as evidence-based guidelines have been developed to investigate its therapeutic use. ${ }^{8}$

Another non-invasive technique that can modulate neuronal function is transcranial direct current stimulation (tDCS). ${ }^{14}$ Its functioning is based on weak electrical currents that are applied over the scalp through electrodes for a specific amount of time. Usually, one electrode is placed over the target region, while a second electrode is placed over a reference area in order to create a closed circuit. Depending on the electrodes, the stimulation can be anodal (producing membrane depolarization, lowering firing thresholds, and consequently increasing neuronal firing) or cathodal (producing membrane hyperpolarization and consequently decreasing neuronal firing). Similar to TMS, tDCS can be used for different purposes, including the management of chronic pain conditions. Moreover, evidence-based guidelines have also been established for the therapeutic use of tDCS. ${ }^{15}$

The effectiveness of these two techniques for the management of chronic pain has been assessed comprehensively in neuropathic pain disorders or fibromyalgia. ${ }^{16-18}$ However, the trigeminal nerve has a number of distinct structures and associated specializations that are not present in spinal nerve distributions. These include innervation of all the special senses, greater percentage of C-fibers than A-delta fibers in some tissue (ie, pulp of teeth), elevated coalescence between sensory and motor roots, and greater spatial degree of peripheral integration, among others. ${ }^{19-21}$ Therefore, the anatomical and functional differences of the trigeminal nerve make OFP disorders less comparable with other disorders presenting in spinal territories.

Although other reviews have assessed the efficacy of noninvasive brain stimulation in different headache disorders, ${ }^{22,23}$ no comprehensive evaluation has been performed in other chronic OFP disorders. Thus, the aim of this systematic review is to assess the efficacy of active TMS and tDCS to reduce pain intensity in different chronic OFP disorders when compared to sham (inactive) stimulation. We also aimed to report relief duration, associated adverse effects, and describe different methodologies used.

\section{Methods}

The review protocol was registered in the International Prospective Register for Systematic Reviews (PROSPERO, protocol CRD42018085762). Recommendations of the Preferred Reporting Items for Systematic reviews and MetaAnalyses (PRISMA) statement were followed. ${ }^{24,25}$ Eligibility criteria were as follows:

1) Population: adult patients ( $>18$ years old) diagnosed with temporomandibular disorders (TMDs), burning mouth syndrome (BMS), persistent dentoalveolar pain (PDAP), atypical facial pain, trigeminal neuralgia (TN), postherpetic neuralgia, trigeminal neuropathic pain, or trigeminal neuropathy.

2) Intervention: active TMS or tDCS stimulation using any protocol.

3) Comparison: sham TMS or tDCS stimulation.

4) Outcome: primary outcome was clinical pain intensity. Secondary outcomes were duration of pain relief, adverse effects, and methodological parameters. 
Controlled trials were included. Case reports, clinical observations, and animal studies were excluded. There was no restriction for language of publication or publication date. Studies that did not present individualized results for the face were not included.

\section{Search strategy}

Literature search was performed in MEDLINE (Ovid interface) and adapted to Embase, Web of Science, EMB, Scopus, Cochrane Central Register of Controlled Trials, and Google Scholar. Ongoing trials and systematic reviews were searched in Clinical Trial Registry (clinicaltrials.gov) and PROSPERO, respectively. The search process was guided by a trained librarian (NC) from Université de Montréal. A detailed search for MEDLINE is described in Box 1. Additionally, hand search was performed using the references of included studies and reviews about non-invasive brain stimulation for identification of other potentially eligible studies. Results of the search were imported into EndNote X7 (Clarivate Analytics, Philadelphia, PA, USA) for removal of duplicates and reference management. The individual references were exported to a spreadsheet with the following information: authors, year of publication, article title, journal, volume, issue, and abstract.

\section{Screening of references and eligibility assessment}

Based on the titles and the abstracts, two of the authors (AHB and $\mathrm{SG}$ ) screened the references independently. Both of them had previous training in the application of the eligibility criteria using 20 randomly selected references. A calibration procedure was then performed with 50 randomly selected references independently reviewed by both raters, resulting in perfect agreement (Cohen's kappa coefficient $=1.00){ }^{26}$ Then, the rest of the references were screened by the two raters who were blinded to each other. The agreement for the screening process between them was very good (Cohen's kappa coefficient $=0.87$ ). Consensus between the two reviewers was attempted when disagreement occurred, while a third rater (GL) arbitrated when consensus could not be reached. Full-length articles of potential references were obtained in order to assess eligibility, which was also done blindly and compared by the same raters using the same process as previously described for references.

\section{Data extraction and study variables}

The articles that met inclusion criteria were obtained in electronic format. Data extraction was performed individually by one author (AHB) and then revised by a second author (SG). If a disagreement occurred, reconciliation between these two authors was performed. Extracted data were number of patients who received active/sham stimulation, age, number of females, type of TMS/tDCS, brain target area, frequency, intensity of stimulation, number of sessions, number of pulses per session (TMS), duration of stimulation (tDCS), adverse effects, and qualitative main results.

\section{Risk of bias assessment}

Individual risk of bias was assessed independently by two authors (AHB and SG) using the Cochrane Risk of Bias

\section{Box I Ovid MEDLINE search strategy}

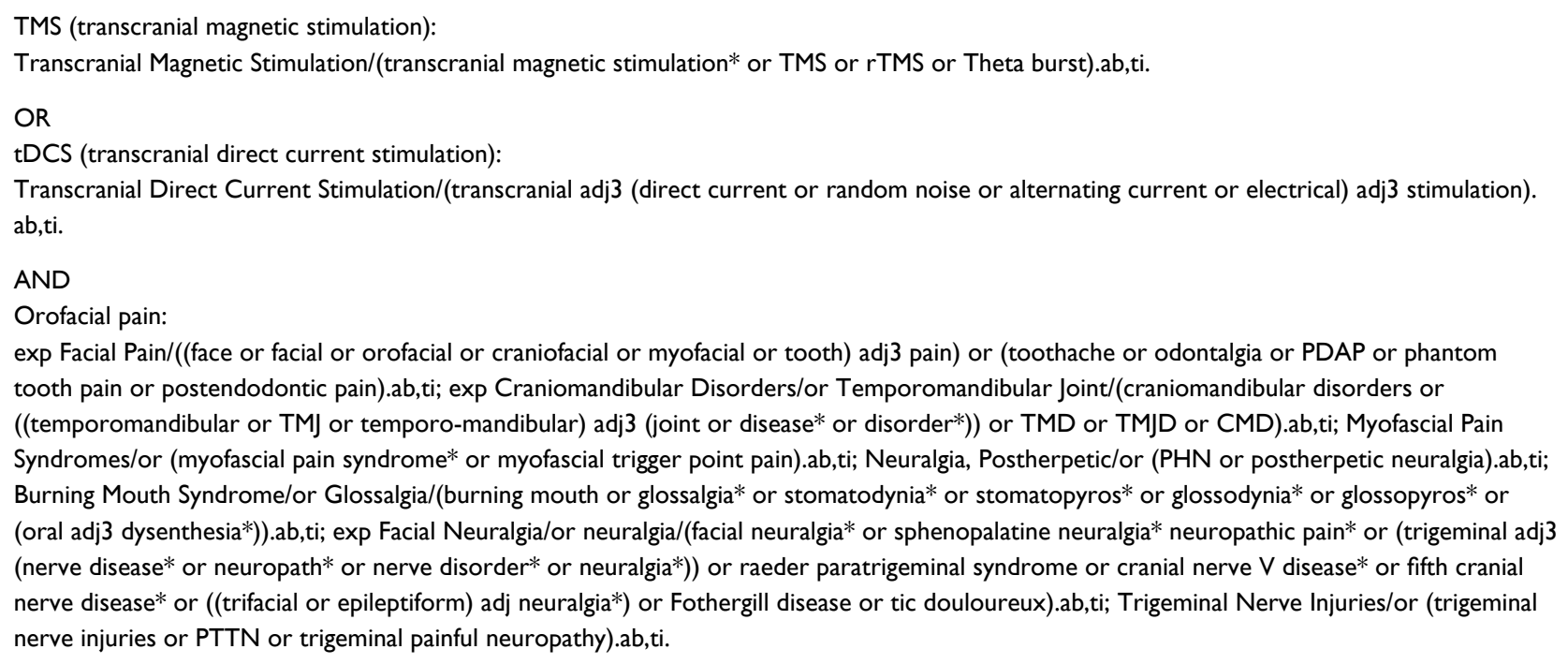


Tool for randomized controlled trials. ${ }^{27}$ If any disagreement occurred, consensus was attempted, and if the disagreement persisted a third author (GL) arbitrated. The following criteria were considered: sequence generation (selection bias), allocation sequence concealment (selection bias), blinding of participants and personnel (performance bias), blinding of outcome assessment (detection bias), incomplete outcome data (attrition bias), selective outcome reporting (reporting bias), and other potential sources of bias. Risk of bias was then classified as low, unclear, or high. Quality of evidence (poor, fair, or good) was obtained by converting these data using the proposed thresholds for Agency for Healthcare Research and Quality (AHRQ) standards.

To reduce publication bias, besides searching reference lists of included articles and systematic reviews about noninvasive brain stimulation, we also searched references in abstracts and posters and contacted corresponding authors if data were missing or were unclear. If they did not reply, the reference was not included in the final selection. As studies were too heterogeneous, a meta-analysis was not performed. Therefore, data are presented in a narrative way.

\section{Results}

\section{Overview of included studies}

Our search generated a total of 556 references from the previously mentioned databases. From those, 29 were selected for full-length text retrieval and 14 met the selection criteria. ${ }^{28-41}$ Hand search of the references from the eligible studies revealed two more studies that were also included in the full-length text retrieval phase but that did not meet the inclusion criteria. Figure 1 depicts a more detailed description of this process using a PRISMA flow diagram. Importantly, ten references were not included as individualized OFP data could not be extracted from the accessible results, which included chronic pain disorders that can be present in the face. Thus, $11 \mathrm{TMS}^{28-38}$ and $3 \mathrm{tDCS}^{39-41}$ studies
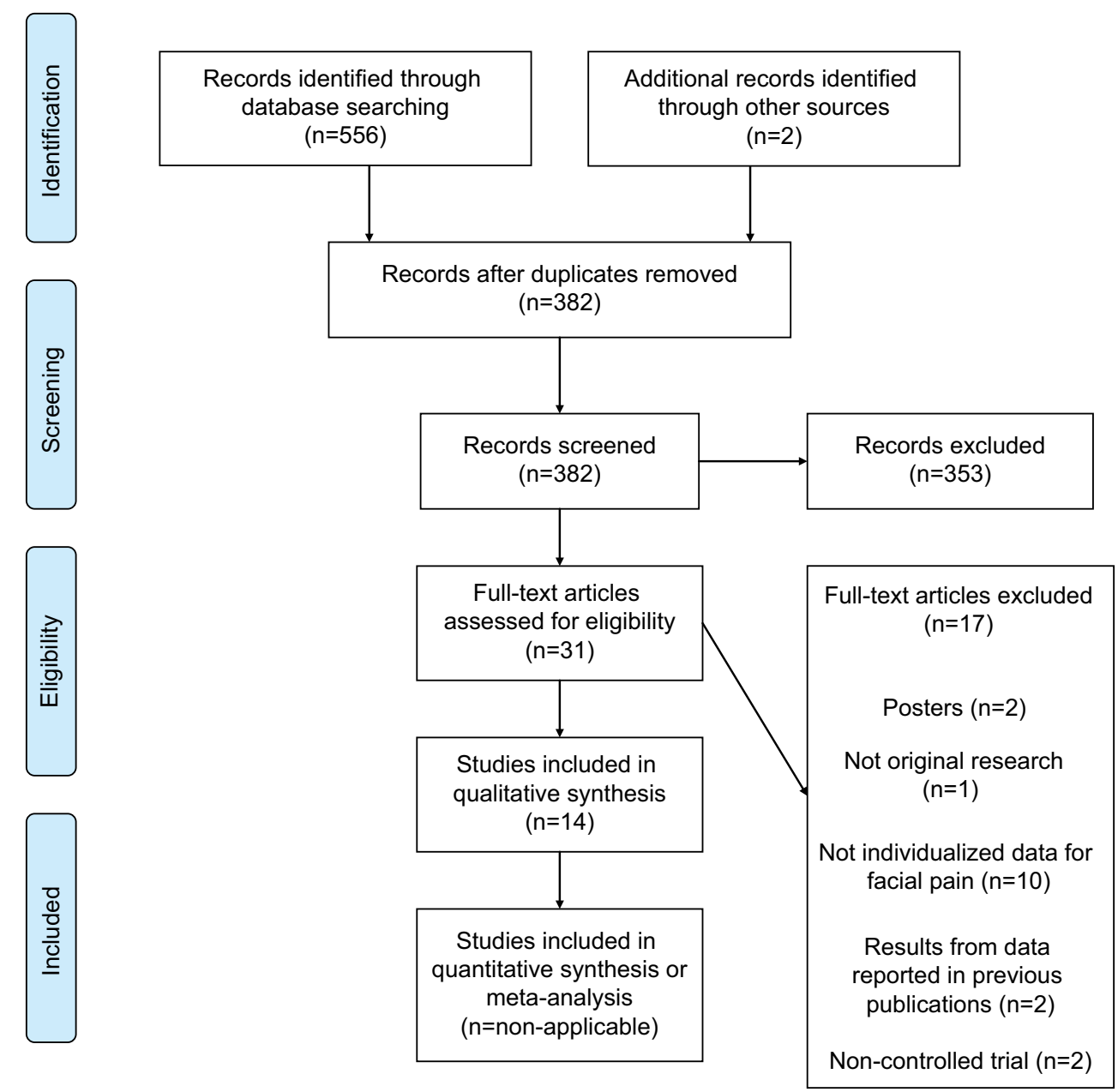

Figure I PRISMA flow diagram. 
were included in this systematic review providing data on a total of 228 patients.

Table 1 summarizes the characteristics, including a description of inclusion and exclusion criteria for each of the 14 studies. Most of the studies were conducted in France $(k=4)$ and the USA $(k=3)$. Six of them had a parallel design, while eight were conducted in a crossover design. It appears that rTMS was used more in neuropathic pain disorders (trigeminal neuropathy and $\mathrm{TN}$ ), conditions with neuropathic features (BMS, PDAP, and related disorders) and not clearly defined, atypical facial pain disorders. Only four rTMS studies were specifically designed for OFP disorders. ${ }^{31,34,37,38}$ The remaining two studies included chronic pain disorders (ie, neuropathic pain) affecting other body areas in addition to the face. In contrast, all tDCS studies were exclusively designed for OFP disorders, exploring the role of tDCS in treating TN or TMD pain.

\section{Methodological parameters and main results}

As the underlying action mechanisms of rTMS are thought to differ from those of tDCS, the results for each technique are reported separately (Tables 2 and 3, respectively). In the 11 rTMS studies, 162 patients were recruited. All of the included studies used high frequency rTMS but one, which used an intermittent theta burst stimulation (iTBS) protocol. ${ }^{34}$ Three of the rTMS studies stimulated different brain areas in a within-subject design and compared the efficacy of the different stimulation locations. Globally, all the studies but one reported significant reductions in pain intensity when compared to sham in at least one of the stimulated areas (Table 3). The targeted brain areas were left prefrontal cortex (LPFC) $[k=2])$, M1 areas corresponding to the hand $(k=4)$ or face $(k=7)$ and right secondary somatosensory and motor cortex (M2/S2) $(k=1)$. Stimulation with rTMS in LPFC, M1 corresponding to the hand, and M2/S2 was associated with significant pain improvement. However, the results after stimulation with rTMS and iTBS in M1 representation of the face were less consistent, with three (3/7) rTMS studies showing no improvement and the iTBS study showing modest and transient relief. Stimulation frequencies for rTMS studies were $5 \mathrm{~Hz}(k=1), 10 \mathrm{~Hz}(k=6)$, and $20 \mathrm{~Hz}$ $(k=3)$, while intensities of stimulation ranged between $80 \%$ and $110 \%$ of the resting motor thresholds. The iTBS study used bursts of $50 \mathrm{~Hz}$ at $90 \%$ of the resting motor threshold for 600 stimulations for one session only. ${ }^{34}$ It also appears that doing multiple sessions of stimulation (5 or 10 sessions) induced more durable analgesic effects, lasting up to
2 weeks in studies using five sessions, ${ }^{31,33}$ and 60 days in a study that used 10 sessions. ${ }^{38}$ Although it was not reported in four studies, the presence of side effects was minimal across the remaining studies, mainly consisting in mild transient headaches $(k=3)$ (Table 2).

For tDCS, 66 patients participated in the three included studies (Table 4). All the studies used anodal tDCS over M1 corresponding to the face. Used intensities were $1 \mathrm{~mA}$ $(k=1)$ and $2 \mathrm{~mA}(k=2)$ for a single 20-min session. Two studies reported significant pain improvement when compared to sham, ${ }^{39,40}$ while a third study, which provided physical therapy in addition to active and sham tDCS, reported pain improvement effects similar to the sham condition. ${ }^{41}$ From those reporting differences, the effects of the intervention lasted up to 4 weeks in one study and 6 weeks in the other one. Minor side effects were present in two of the studies and consisted mainly of mild transient headaches, itching/ tingling, and sleepiness (Table 3).

\section{Risk of bias and quality of studies}

Overall, the quality of the study reporting was poor (12 out of 14) with only two studies being assessed as having fair quality. ${ }^{32,41}$ Therefore, no study presented good reporting quality according to our assessment. High and unclear selection bias, detection bias, and other bias were present in the majority of the studies (Table 4). Although two studies reported no randomization, seven studies reported having performed a randomization strategy but without further specification about the process. Allocation concealment was only reported in two studies. ${ }^{32,41}$ Although blinding of participants was reported in all the studies but one, blinding of outcome assessment was only reported in six studies. Other considered bias included the intake of anticonvulsant medication, which was unclear or high in all the studies but one,${ }^{30}$ and the lack of an appropriate control group. ${ }^{41}$

\section{Discussion}

The results obtained from the studies included in this systematic review indicate that TMS, and in a more limited way tDCS, are safe and effective techniques for reducing patient reported pain intensity when compared to sham stimulation in chronic OFP disorders. These pain reductions were maintained for at least 8 days and up to 60 days in 5 of the 14 studies.

\section{TMS}

The effects ofTMS are influenced by stimulation parameters. These mainly include target brain area (where the magnetic 
Table I Study characteristics

\begin{tabular}{|c|c|c|c|c|c|}
\hline Study & Country & $\begin{array}{l}\text { Study } \\
\text { design }\end{array}$ & OFP condition & Inclusion criteria & Exclusion criteria \\
\hline $\begin{array}{l}\text { Andre-Obadia } \\
\text { et al, } 2018^{28}\end{array}$ & France & Crossover & Trigeminal neuropathy & $\begin{array}{l}\text { Pharmacoresistant neuropathic pain } \\
\text { for I year or longer, stable medication } \\
\text { last month }\end{array}$ & $\begin{array}{l}\text { Epilepsy, addiction, migraine, intracranial } \\
\text { ferromagnetic material, or implanted } \\
\text { stimulator (intracerebral or not, such as } \\
\text { pacemaker) }\end{array}$ \\
\hline $\begin{array}{l}\text { Ayache et al, } \\
2016^{29}\end{array}$ & France & Crossover & $\begin{array}{l}\text { TN after surgery, } \\
\text { atypical facial pain after } \\
\text { dental surgery }\end{array}$ & $\begin{array}{l}\text { Chronic refractory unilateral } \\
\text { neuropathic pain, stable medication }\end{array}$ & $\begin{array}{l}\text { No contraindications to magnetic } \\
\text { stimulation, including no history of } \\
\text { epilepsy and/or ferromagnetic implant }\end{array}$ \\
\hline $\begin{array}{l}\text { Borckardt } \\
\text { et al, } 2009^{30}\end{array}$ & USA & Crossover & $\begin{array}{l}\text { Trigeminal neuropathy, } \\
\text { atypical facial pain }\end{array}$ & $\begin{array}{l}\text { Chronic neuropathic pain for I year } \\
\text { or longer }\end{array}$ & $\begin{array}{l}\text { Family history of epilepsy, seizures, } \\
\text { implanted devices, history of tumors or } \\
\text { brain abnormalities, implanted metal in } \\
\text { the head, neck, or chest, and medications } \\
\text { that lower seizure threshold }\end{array}$ \\
\hline $\begin{array}{l}\text { Fricova et al, } \\
2013^{31}\end{array}$ & $\begin{array}{l}\text { Czech } \\
\text { Republic }\end{array}$ & Parallel & $\begin{array}{l}\text { TN after dental or } \\
\text { neural surgery, atypical } \\
\text { facial pain }\end{array}$ & $\begin{array}{l}\text { 18-65-year-old patients with } \\
\text { pharmacoresistant }{ }^{\mathrm{a}} \text { facial pain for } \\
6 \text { months or longer while on stable } \\
\text { analgesic medication }\end{array}$ & $\begin{array}{l}\text { Severe organic brain damage, serious } \\
\text { diseases, epilepsy, or metallic implants in } \\
\text { the body }\end{array}$ \\
\hline $\begin{array}{l}\text { Hosomi et al, } \\
2013^{32}\end{array}$ & Japan & Crossover & $\begin{array}{l}\text { Trigeminal neuropathic } \\
\text { pain }\end{array}$ & $\begin{array}{l}\geq 20 \text {-year-old patients with } \\
\text { neuropathic pain per IASP criteria } \\
\text { for more than } 6 \text { months, stable } \\
\text { medications }\end{array}$ & $\begin{array}{l}\text { Inability to fill the questionnaires, } \\
\text { dementia, aphasia, major psychiatric } \\
\text { disease, suicidal wish, pregnancy, } \\
\text { and contraindications to TMS, like } \\
\text { implantation of a cardiac pacemaker }\end{array}$ \\
\hline $\begin{array}{l}\text { Khedr et al, } \\
2005^{33}\end{array}$ & Egypt & Parallel & $\mathrm{TN}$ & TN diagnosis as IASP criteria & $\begin{array}{l}\text { Intracranial metallic devices, pacemakers } \\
\text { or any device, extensive myocardial } \\
\text { ischemia, and epilepsy }\end{array}$ \\
\hline $\begin{array}{l}\text { Kohutova et al, } \\
2017^{34}\end{array}$ & $\begin{array}{l}\text { Czech } \\
\text { Republic }\end{array}$ & Parallel & Chronic OFP & $\begin{array}{l}\text { I8-65-year-old patients with } \\
\text { pharmacoresistant }{ }^{\mathrm{a}} \text { facial pain for } \\
6 \text { months or longer while on stable } \\
\text { analgesic medication }\end{array}$ & $\begin{array}{l}\text { Severe organic brain damage, serious } \\
\text { diseases, epilepsy, or metallic implants in } \\
\text { the body }\end{array}$ \\
\hline $\begin{array}{l}\text { Lefaucheur } \\
\text { et al, 200I }{ }^{35}\end{array}$ & France & Crossover & $\begin{array}{l}\text { Trigeminal neuropathy } \\
\text { after surgery }\end{array}$ & $\begin{array}{l}\text { Chronic unilateral pharmacoresistant } \\
\text { neuropathic pain }\end{array}$ & History of seizures \\
\hline $\begin{array}{l}\text { Lefaucheur } \\
\text { et al, } 2004^{36}\end{array}$ & France & Crossover & $\begin{array}{l}\text { Trigeminal nerve lesion } \\
\text { (failure of } \mathrm{TN} \text { surgery) }\end{array}$ & $\begin{array}{l}\text { Chronic unilateral pharmacoresistant } \\
\text { neurogenic pain }\end{array}$ & History of seizures \\
\hline $\begin{array}{l}\text { Lindholm et al, } \\
2015^{37}\end{array}$ & Finland & Crossover & $\begin{array}{l}\text { Trigeminal neuropathic } \\
\text { pain, atypical facial pain, } \\
\text { and BMS }\end{array}$ & $\begin{array}{l}\text { Chronic daily neuropathic pain as per } \\
\text { ICHD } 2013 \text {, with } 4 / 10 \text { pain intensity, } \\
\text { stable medication }\end{array}$ & $\begin{array}{l}\text { History of seizure, pacemaker } \\
\text { implantation, major stroke, or other } \\
\text { contra-indication for TMS }\end{array}$ \\
\hline $\begin{array}{l}\text { Umezaki et al, } \\
2016^{38}\end{array}$ & USA & Parallel & BMS & $\begin{array}{l}\text { Daily deep bilateral burning sensation } \\
\text { of oral mucosa at least } 4-6 \text { months, } \\
\text { constant/increasing intensity during } \\
\text { day, no worsening but possible } \\
\text { improvement on eating/drinking, no } \\
\text { interference with sleep and normal } \\
\text { aspect. Stable medications }\end{array}$ & $\begin{array}{l}\text { Evident inflammation or autoimmune } \\
\text { disease, current primary psychiatric } \\
\text { condition, history of substance abuse, } \\
\text { history of seizures, brain surgery, } \\
\text { intracranial hypertension, pace-maker } \\
\text { or other metallic implants, medication } \\
\text { changes within } 4 \text { weeks of starting or } \\
\text { during the trial }\end{array}$ \\
\hline $\begin{array}{l}\text { Donnell et al, } \\
2015^{39}\end{array}$ & USA & Parallel & TMD (myofascial) & $\begin{array}{l}\text { I8-65-year-old patients with daily } \\
\text { chronic myofascial TMD based on } \\
\text { RDC/TMD, not adequately controlled } \\
\text { by conventional therapy for a year, } \\
\text { surgery naïve, pain } 3 / 102 \text { weeks } \\
\text { before the study, and stable medication }\end{array}$ & $\begin{array}{l}\text { Pain not primarily due to myofascial } \\
\text { TMS, history of current evident } \\
\text { neurological disorder (epilepsy, major } \\
\text { depression, stroke, neuropathy, or } \\
\text { neuropathic pain) }\end{array}$ \\
\hline $\begin{array}{l}\text { Hagenacker } \\
\text { et al, } 2014^{40}\end{array}$ & Germany & Crossover & $\mathrm{TN}$ & $\begin{array}{l}\text { Classic TN with/without concomitant } \\
\text { persistent pain per ICDH3, last } \\
6 \text { months stable medication }\end{array}$ & $\begin{array}{l}\text { Other pain disorders and other } \\
\text { disorders of the nervous system }\end{array}$ \\
\hline $\begin{array}{l}\text { Oliveira et al, } \\
2015^{41}\end{array}$ & Brazil & Parallel & TMD (myofascial) & $\begin{array}{l}\text { Patients with myofascial pain or } \\
\text { myofascial with limited opening per } \\
\text { RDC/TMD with } 4 / 10 \text { intensity in the } \\
\text { last } 6 \text { months }\end{array}$ & $\begin{array}{l}\text { Physical therapy in the last month, } \\
\text { rheumatic or cardiovascular disease, } \\
\text { metal implant in the brain or skull }\end{array}$ \\
\hline
\end{tabular}

Note: aPharmacoresistant defined as persistence of pain, despite at least two attempts at pharmacological treatment in the past, both of sufficient dose and sufficient time. Abbreviations: BMS, burning mouth syndrome; ICHD, International Classification of Headache Disorders; IASP, International Association for the Study of Pain; OFP, orofacial pain; TMD, temporomandibular disorders; TMS, transcranial magnetic stimulation; TN, trigeminal neuralgia; RDC, research diagnostic criteria. 


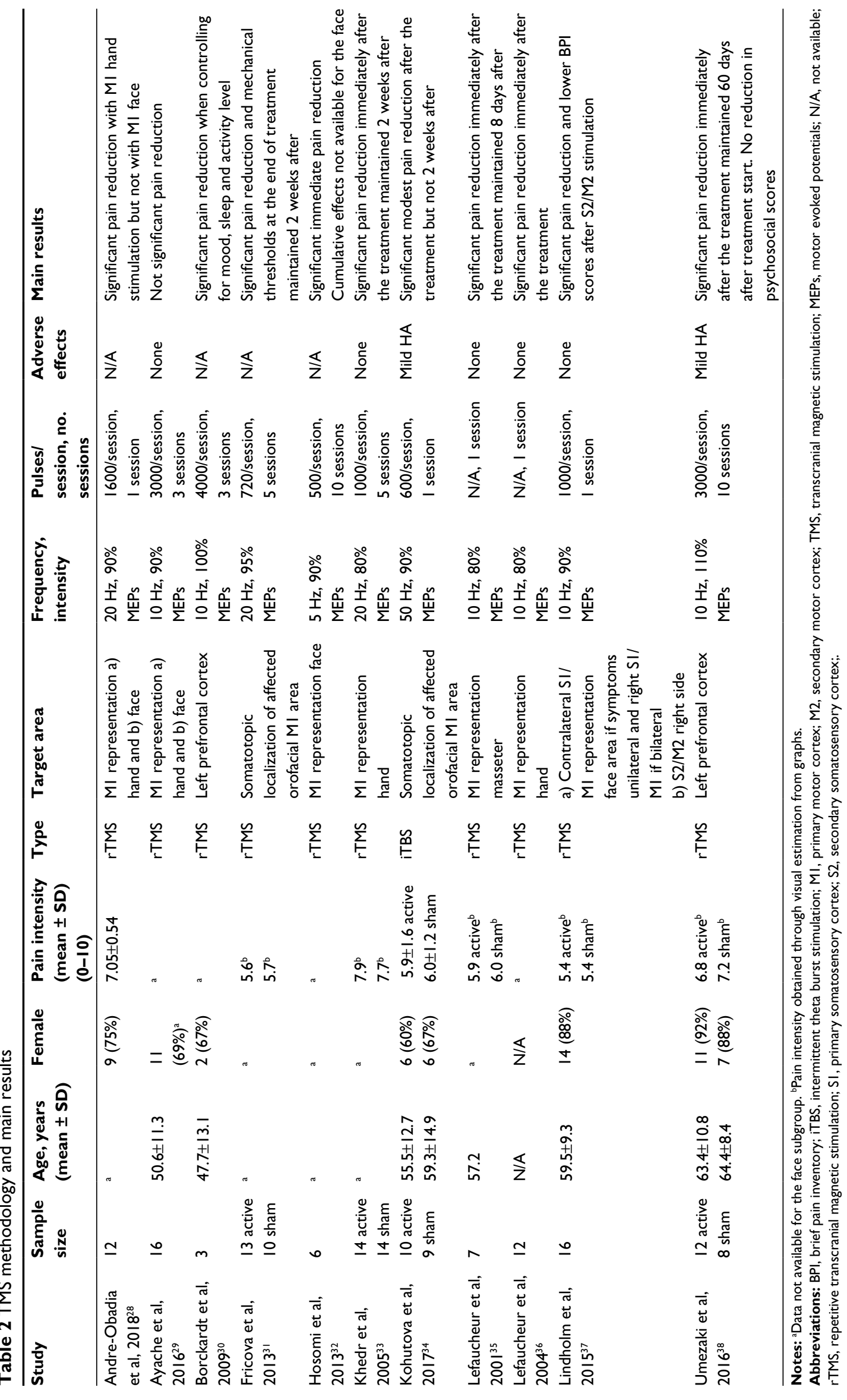


coil is placed), stimulation intensity and frequency, the number of pulses delivered per session, and the number of sessions that are performed. Currently, there is no consensus about the ideal methodology or protocol to follow in chronic pain treatment. However, the need of standardizing the report of outcomes, reducing the risk of bias, and increasing research quality has been highlighted in order to allow better comparisons and to be able to define optimal stimulation parameters. $^{42}$

The stimulation of M1 with high frequencies ( $\geq 5 \mathrm{~Hz}$ ) of rTMS has been determined to have a definite analgesic effect (level A of evidence) in neuropathic pain, and its use is recommended for treating pain disorders. ${ }^{8}$ Nevertheless, a question arises as to what area of M1 should be stimulated: if a) the somatotopic representation of the face area or b) the somatotopic representation of hand area, which has been shown to decrease pain in other areas than the hand when used in "non-diffuse" neuropathic pain disorders. ${ }^{43,44}$ The results of the present review showed that while the stimulation of the hand area led to significant pain intensity reduction effects in the majority of the included studies, stimulation of the face area was associated with more inconsistent results in OFP (in three of the six studies, no improvement was reported) (Table 2). Although speculative, authors ${ }^{28}$ have proposed three main arguments to explain this discrepancy in the results: a) the difficulty to determine the "hot spot" and resting motor thresholds in the face muscles when compared to the hand; b) the higher connectivity pattern of M1 representation of the hand relative to the M1 representation of the face with brain structures related with endogenous pain modulation; and c) the reduction of power due to the presence of thicker layers of cerebral spinal fluid (CSF) in proximal regions of the M1 representation of the face. Whatever it may be, findings from the present systematic review seem to favor the application of TMS toward the hand M1 area rather than the face to treat OFP disorders.

This review also showed that stimulation over the LPFC produced significant decreases in pain intensity in disorders such as BMS or neuropathic pains. The stimulation of this area has been shown to be effective in managing depressive symptoms, which are frequently present in chronic pain conditions, and to be effective in some fibromyalgia studies. ${ }^{45,46}$ Interestingly, Borckardt et al showed that the induced analgesic effects were independent from mood scores in three neuropathic pain patients. ${ }^{30}$ However, the pilot nature of this study warrants caution when interpreting these conjectural findings.

Right S2/M2 has also been investigated in one study having positive results. ${ }^{37}$ The authors hypothesized that obtained 
Table 4 Risk of bias assessed by Cochrane Risk of Bias Tool for Randomized Controlled Trials

\begin{tabular}{|c|c|c|c|c|c|c|c|c|}
\hline Study & $\begin{array}{l}\text { Random } \\
\text { sequence } \\
\text { generation }\end{array}$ & $\begin{array}{l}\text { Allocation } \\
\text { concealment }\end{array}$ & $\begin{array}{l}\text { Selective } \\
\text { reporting }\end{array}$ & $\begin{array}{l}\text { Blinding } \\
\text { participants } \\
\text { and } \\
\text { personnel }\end{array}$ & $\begin{array}{l}\text { Blinding } \\
\text { outcome } \\
\text { assessment }\end{array}$ & $\begin{array}{l}\text { Incomplete } \\
\text { outcome } \\
\text { data }\end{array}$ & $\begin{array}{l}\text { Other } \\
\text { bias }\end{array}$ & $\begin{array}{l}\text { Overall } \\
\text { quality }\end{array}$ \\
\hline Andre-Obadia et al, $2018^{28}$ & Unclear & High & Low & Low & High & Low & Unclear & Poor \\
\hline Ayache et al, $2016^{29}$ & High & High & Low & Low & High & Low & High & Poor \\
\hline Borckardt et al, $2009^{30}$ & Unclear & High & Low & Low & High & Low & Low & Poor \\
\hline Fricova et al, $2013^{31}$ & Unclear & Unclear & Low & Low & Low & Low & High & Poor \\
\hline Hosomi et al, $2013^{32}$ & Low & Low & Low & Low & Low & Low & High & Fair \\
\hline Khedr et al, $2005^{33}$ & High & High & Low & Low & Low & Low & Unclear & Poor \\
\hline Kohutova et al, $2017^{34}$ & Low & Unclear & Low & Low & Low & Low & Unclear & Poor \\
\hline Lefaucheur et al, 200I 35 & Unclear & High & Low & High & High & Low & Unclear & Poor \\
\hline Lefaucheur et al, $2004^{36}$ & Unclear & High & Low & Low & High & Low & Unclear & Poor \\
\hline Lindholm et al, $2015^{37}$ & Unclear & High & Low & Low & High & Low & Unclear & Poor \\
\hline Umezaki et al, $2016^{38}$ & Low & Unclear & Low & Low & High & Low & High & Poor \\
\hline Donnell et al, $2015^{39}$ & Low & Unclear & Low & Low & High & Low & Unclear & Poor \\
\hline Hagenacker et al, $2014^{40}$ & Unclear & High & Low & Low & Low & Low & High & Poor \\
\hline Oliveira et al, 2015 $5^{4 I}$ & Low & Low & Low & Low & Low & Low & High & Fair \\
\hline
\end{tabular}

analgesic effects may be due to the proximity of this area to anatomical areas that play important roles in pain perception, known as the "pain matrix" 47 and the high degree of connections observed in $\mathrm{S} 2$ and other brain locations during painful stimulation. ${ }^{48}$ Therefore, LPFC and S2/M2 areas could be considered as alternative target areas to M1 for the investigation of rTMS in chronic OFP.

According to our results, it appears that more sessions and more pulses per session were associated with longer-lasting effects. The study of Umezaki et al, ${ }^{38}$ which used the 3,000 pulses per session and the most number of sessions (10 sessions), showed most durable effects ( 60 days after beginning of treatment). The latter study also stimulated at the highest intensity ( $110 \%$ of resting motor thresholds) with very few adverse effects. Two independent studies by Khedr et $\mathrm{al}^{33}$ and Fricova et $\mathrm{a}^{31}$ showed analgesic effects that outlasted 14 days with five rTMS sessions, suggesting that more sessions and pulses delivered are associated with more durable analgesic effects. All the included studies used rTMS protocols but one, which used iTBS. ${ }^{34}$ This technique is an adaptation of high frequency rTMS, ${ }^{11}$ and a protocol with bursts of 3-5 pulses at $50 \mathrm{~Hz}$ in intraburst and $5 \mathrm{~Hz}$ in interburst was used to excite neurons. However, evidence with regard to its possible use in OFP is very limited, as only one study used this protocol in OFP, showing modest and transient effects. ${ }^{34}$

In regard to its safety, rTMS seems to be a safe technique as only minor and transient adverse effects were reported in a minority of included studies. In fact, only two studies reported mild headaches, and five reported no incidence of any adverse effect. It is also important to mention that data related to safety were not available in four of the included studies.

\section{tDCS}

Regarding the use of tDCS in OFP treatment, data were more limited with only three studies being included in our review, which limits the interpretation of the results. Two of them assessed its use in TMD patients, while the other explored it in TN. As in rTMS, the stimulation protocols were not standardized across studies, although less methodological differences have usually been observed relative to rTMS studies in chronic pain. ${ }^{15}$ Although some studies stimulated areas like LFPC or primary visual cortex (V1) in the case of migraines, the $\mathrm{M} 1$ region seems the most common target for chronic pain treatment using anodal stimulation. ${ }^{15}$ This was also the case in the present systematic review, where all the included studies targeted M1. It has been determined that anodal stimulation of the left M1 (commonly used for more diffuse pains) has a probable effect on pain reduction in fibromyalgia (level $\mathrm{B}$ of evidence), but its use in neuropathic pain (level $\mathrm{C}$ in spinal cord lesion, and no recommendation in peripheral) is more debated. ${ }^{15}$ Interestingly, in TN patients, tDCS reduced pain intensity in the purely paroxysmal subgroup and not in TN patients with a concomitant diffuse background pain. ${ }^{40}$ The parameters and methodologies for stimulation were in line with the literature corresponding to spinally mediated neuropathic pain and fibromyalgia. Adverse effects were also minimal and only reported in two of the studies. These included mild headaches, tingling, and sleepiness, which were also reported in placebo groups. 
As presented in this review, there is less tDCS data available when compared to rTMS to assess its use in OFP or other pain conditions. ${ }^{15}$ More evidence supports the use of rTMS for treating chronic pain, but some elements need to be considered. It has been speculated that rTMS may be more effective managing pain with neuropathic characteristics located in the upper limbs and face, while tDCS appears to be more effective treating musculoskeletal pain conditions such as fibromyalgia and in a minor way neuropathic pain located in the lower limb due to spinal cord lesion. ${ }^{8,15,49}$ This observation may be the reason why included rTMS studies were performed mostly in OFP disorders with neuropathic characteristics, while TMDs were only studied with tDCS.

\section{Risk of bias and quality of the studies}

According to our assessment, there was a considerable risk of bias present in all the included studies across different categories (Table 4 ), thus making the overall quality of the including studies fair $(k=2)$ or poor in its vast majority $(k=12)$. The lack of description and clarity of the randomization process, the absence of a concealment method or its omission in the report, and the non-blinding in the assessment of outcomes were some of the issues that increased the risk of bias. It also needs to be mentioned that we decided to assess the intake of anticonvulsants or GABAergic medications as another possible bias in the category of other risk of bias. The use of stable medication was allowed in the majority of the studies..$^{28,29,31,32,34,38-40}$ Some of the medications to manage chronic OFP disorders, especially the ones with neuropathic characteristics, include anticonvulsants (sodium and calcium channel blockers) and GABA agonists among others. ${ }^{50}$ Although the interruption of these medications when stable can suppose an ethical concern and may increase the risk of TMS-induced seizures, ${ }^{7}$ it has been suggested that these medications can alter treatment outcomes and add noise to the therapeutic effects of rTMS and anodal tDCS, ${ }^{42,51-56}$ possibly compromising its optimal efficacy. Even though Ayache et al controlled for the possible influence of medications in active/ sham stimulations in their analyses, ${ }^{29}$ only one study excluded participants taking this type of medications. ${ }^{30}$ However, the latter was a pilot study where only four participants (three of them with facial pain) were included. Therefore, despite not being clinically representative, investigating the effects of rTMS and tDCS in the absence of these medications may lead to different results. Another interesting route with more clinical meaning would be to investigate the effects of these neurostimulation techniques in the possible reduction of pain medication intake or drug sparing. Different studies have shown positive effects of both techniques in reducing opioid consumption in patients with substance abuse disorders or postsurgical pain. ${ }^{57-59}$ As the medications used to treat chronic pain are frequently associated with multiple side effects, drug interactions, and safety issues, ${ }^{50,60}$ the use of non-invasive stimulation to reduce its intake surface as appealing. Quantifying the medication intake with scales such as Medication Quantification Scale (MQS) ${ }^{61}$ before and after these interventions can be considered as an interesting outcome to explore. In addition, despite grey literature was searched in order to reduce it, the possible risk of publication bias also needs to be mentioned. ${ }^{62}$ In conclusion and in line with recent literature, ${ }^{42}$ our risk of bias assessment reinforces the need of improving the quality of rTMS and tDCS studies by reducing the possible sources of bias mentioned above.

Considerable heterogeneity was observed across studies. First, as this review aimed to be as inclusive as possible with regard to OFP disorders, disorders with different pathophysiological mechanisms and etiologies were studied, so the comparison between the results of these studies was somewhat difficult. In addition: a) some of the studies used a "flexible" inclusion criteria that included per se disorders with different pain characteristics; ${ }^{29-31,37}$ b) others did not report a specific diagnosis $;{ }^{34}$ and c) others were not specifically focused on OFP but in pain conditions that could present in the face (ie, neuropathic pain), ${ }^{28,29,32,35,36}$ making data extraction and interpretation quite complex. Second, outcomes also varied considerably between studies. Pain intensity was reported in different manners, including means or individual data of visual analog scale (VAS) or numerical rating scale (NRS), or different formulas calculating percentages of reduction (including or not sham reduction). ${ }^{32}$ And third, the report and application of rTMS sham protocols, which are important to assess pain improvement in a controlled design, ${ }^{42,63}$ also varied considerably among the included studies. These limitations and the low number of tDCS studies prevented us from considering the possibility of performing a meta-analysis, leading us to present the data in a more narrative manner.

\section{Limitations}

This systematic review has some limitations. As explained before, the quality of the included studies is not ideal, and inclusion of different OFP disorders makes our results less specific and broader. In addition, the exclusion of headache disorders affecting the orofacial region such as migraine or trigeminal autonomic cephalalgias (TACs) may be considered a limitation. However, considering that a) the inclusion of headaches in OFP disorders classifications still remains 
controversial; ${ }^{64} \mathrm{~b}$ ) systematic reviews and meta-analysis have already been performed in TMS/tDCS and migraine; ${ }^{22,65}$ and c) TACs are very rare disorders, ${ }^{66}$ we decided not to include headache disorders in our review. Finally, it is also important to mention that we did not assess in detail the quality and difference of applied sham protocols or the type of coil that was used.

\section{Conclusion}

This review assessed the use of non-invasive brain stimulation (TMS and tDCS) in chronic OFP disorders. Although TMS appears to be a safe and promising alternative to reduce pain intensity in different chronic OFP disorders, evidence is still quite limited, especially with regard to tDCS. Additional research effort is needed to characterize optimal brain stimulation parameters in order to promote TMS/tDCS immediate and long-term efficacy for treatment of chronic OFP disorders. Standardization of reported outcomes, homogeneity of samples, and reduction of bias are necessary to improve the quality of the studies and promote a better analysis of their results.

\section{Acknowledgments}

The authors would like to acknowledge Natalie Clairoux from Université de Montréal for her guidance and Estephan J. Moana Filho, DDS, MS, PhD, from University of Minnesota for his contributions.

\section{Disclosure}

The authors report no conflicts of interest in this work.

\section{References}

1. Benoliel R, Sharav Y. Chronic orofacial pain. Curr Pain Headache Rep. 2010;14(1):33-40.

2. Lipton JA, Ship JA, Larach-Robinson D. Estimated prevalence and distribution of reported orofacial pain in the United States. J Am Dent Assoc. 1993;124(10):115-121.

3. Reisine ST, Weber J. The effects of temporomandibular joint disorders on patients' quality of life. Community Dent Health. 1989;6(3):257-270.

4. Shueb SS, Nixdorf DR, John MT, Alonso BF, Durham J. What is the impact of acute and chronic orofacial pain on quality of life? J Dent. 2015;43(10):1203-1210.

5. Chronic Pain Research Alliance. Impact of Chronic Overlapping Pain Conditions on Public Health and the Urgent Need for Safe and Effective Treatment. 2015:1-46. Available from: http://www.chronicpainresearch. org/public/CPRA_WhitePaper_2015-FINAL-Digital.pdf. Accessed August 17, 2016.

6. Barker AT, Jalinous R, Freeston IL. Non-invasive magnetic stimulation of human motor cortex. Lancet. 1985;1(8437):1106-1107.

7. Rossi S, Hallett M, Rossini PM, Pascual-Leone A; Safety of TMS Consensus Group. Safety, ethical considerations, and application guidelines for the use of transcranial magnetic stimulation in clinical practice and research. Clin Neurophysiol. 2009;120(12):2008-2039.
8. Lefaucheur JP, Andre-Obadia N, Antal A, et al. Evidence-based guidelines on the therapeutic use of repetitive transcranial magnetic stimulation (rTMS). Clin Neurophysiol. 2014;125(11):2150-2206.

9. Nguyen JP, Nizard J, Keravel Y, Lefaucheur JP. Invasive brain stimulation for the treatment of neuropathic pain. Nat Rev Neurol. 2011;7(12):699-709.

10. DosSantos MF, Ferreira N, Toback RL, Carvalho AC, DaSilva AF. Potential mechanisms supporting the value of motor cortex stimulation to treat chronic pain syndromes. Front Neurosci. 2016;10:18.

11. Huang YZ, Edwards MJ, Rounis E, Bhatia KP, Rothwell JC. Theta burst stimulation of the human motor cortex. Neuron. 2005;45(2):201-206.

12. Di Lazzaro V, Pilato F, Dileone M, et al. The physiological basis of the effects of intermittent theta burst stimulation of the human motor cortex. J Physiol. 2008;586(16):3871-3879.

13. Jodoin M, Rouleau D, Larson-Dupuis C, Gosselin N, De Beaumont L. The clinical utility of repetitive transcranial magnetic stimulation in reducing the risks of transitioning from acute to chronic pain in traumatically injured patients. Prog Neuropsychopharmacol Biol Psychiatry. Epub 2017 Jul 8.

14. Nitsche MA, Paulus W. Excitability changes induced in the human motor cortex by weak transcranial direct current stimulation. J Physiol. 2000;527(pt 3):633-639.

15. Lefaucheur JP, Antal A, Ayache SS, et al. Evidence-based guidelines on the therapeutic use of transcranial direct current stimulation (tDCS). Clin Neurophysiol. 2017;128(1):56-92.

16. Lefaucheur JP. The use of repetitive transcranial magnetic stimulation (rTMS) in chronic neuropathic pain. Neurophysiol Clin. 2006;36(3):117-124.

17. Saltychev M, Laimi K. Effectiveness of repetitive transcranial magnetic stimulation in patients with fibromyalgia: a meta-analysis. Int J Rehabil Res. 2017;40(1):11-18.

18. O'Connell NE, Wand BM, Marston L, Spencer S, DeSouza LH. Non-invasive brain stimulation techniques for chronic pain. Cochrane Database Syst Rev. 2014;(4):CD008208.

19. Sessle BJ. Neural mechanisms and pathways in craniofacial pain. Can J Neurol Sci. 1999;26(suppl 3):S7-S11.

20. Sessle BJ. Peripheral and central mechanisms of orofacial inflammatory pain. Int Rev Neurobiol. 2011;97:179-206.

21. Bereiter DA, Hirata H, Hu JW. Trigeminal subnucleus caudalis: beyond homologies with the spinal dorsal horn. Pain. 2000;88(3):221-224.

22. Shirahige L, Melo L, Nogueira F, Rocha S, Monte-Silva K. Efficacy of noninvasive brain stimulation on pain control in migraine patients: a systematic review and meta-analysis. Headache. 2016;56(10):1565-1596.

23. Zhu S, Marmura MJ. Non-invasive neuromodulation for headache disorders. Curr Neurol Neurosci Rep. 2016;16(2):11.

24. Liberati A, Altman DG, Tetzlaff J, et al. The PRISMA statement for reporting systematic reviews and meta-analyses of studies that evaluate health care interventions: explanation and elaboration. PLoS Med. 2009;6(7):e1000100.

25. Moher D, Liberati A, Tetzlaff J, Altman DG; PRISMA Group. Preferred reporting items for systematic reviews and meta-analyses: the PRISMA statement. PLoS Med. 2009;6(7):e1000097.

26. Cohen J. A coefficient of agreement for nominal scales. Educ Psychol Meas. 1960;20(1):37-46.

27. Higgins JP, Altman DG, Gotzsche PC, et al. The Cochrane Collaboration's tool for assessing risk of bias in randomised trials. $B M J$. 2011;343:d5928.

28. Andre-Obadia N, Magnin M, Simon E, Garcia-Larrea L. Somatotopic effects of rTMS in neuropathic pain? A comparison between stimulation over hand and face motor areas. Eur J Pain. 2018;22(4):707-715.

29. Ayache SS, Ahdab R, Chalah MA, et al. Analgesic effects of navigated motor cortex rTMS in patients with chronic neuropathic pain. Eur $J$ Pain. 2016;20(9):1413-1422.

30. Borckardt JJ, Smith AR, Reeves ST, et al. A pilot study investigating the effects of fast left prefrontal rTMS on chronic neuropathic pain. Pain Med. 2009;10(5):840-849. 
31. Fricova J, Klirova M, Masopust V, Novak T, Verebova K, Rokyta R. Repetitive transcranial magnetic stimulation in the treatment of chronic orofacial pain. Physiol Res. 2013;62(suppl 1):S125-S134.

32. Hosomi K, Shimokawa T, Ikoma K, et al. Daily repetitive transcranial magnetic stimulation of primary motor cortex for neuropathic pain: a randomized, multicenter, double-blind, crossover, sham-controlled trial. Pain. 2013;154(7):1065-1072.

33. Khedr EM, Kotb H, Kamel NF, Ahmed MA, Sadek R, Rothwell JC. Longlasting antalgic effects of daily sessions of repetitive transcranial magnetic stimulation in central and peripheral neuropathic pain. J Neurol Neurosurg Psychiatry. 2005;76(6):833-838.

34. Kohutova B, Fricova J, Klirova M, Novak T, Rokyta R. Theta burst stimulation in the treatment of chronic orofacial pain: a randomized controlled trial. Physiol Res. 2017;66(6):1041-1047.

35. Lefaucheur JP, Drouot X, Nguyen JP. Interventional neurophysiology for pain control: duration of pain relief following repetitive transcranial magnetic stimulation of the motor cortex. Neurophysiol Clin. 2001;31(4):247-252.

36. Lefaucheur JP, Drouot X, Menard-Lefaucheur I, et al. Neurogenic pain relief by repetitive transcranial magnetic cortical stimulation depends on the origin and the site of pain. J Neurol Neurosurg Psychiatry. 2004;75(4):612-616.

37. Lindholm P, Lamusuo S, Taiminen T, et al. Right secondary somatosensory cortex-a promising novel target for the treatment of drug-resistant neuropathic orofacial pain with repetitive transcranial magnetic stimulation. Pain. 2015;156(7):1276-1283.

38. Umezaki Y, Badran BW, DeVries WH, Moss J, Gonzales T, George MS. The efficacy of daily prefrontal repetitive transcranial magnetic stimulation (rTMS) for burning mouth syndrome (BMS): a randomized controlled single-blind study. Brain Stimulat. 2016;9(2):234-242.

39. Donnell A, D Nascimento T, Lawrence M, et al. High-definition and non-invasive brain modulation of pain and motor dysfunction in chronic TMD. Brain Stimul. 2015;8(6):1085-1092.

40. Hagenacker T, Bude V, Naegel S, et al. Patient-conducted anodal transcranial direct current stimulation of the motor cortex alleviates pain in trigeminal neuralgia. $J$ Headache Pain. 2014;15:78.

41. Oliveira LB, Lopes TS, Soares C, et al. Transcranial direct current stimulation and exercises for treatment of chronic temporomandibular disorders: a blind randomised-controlled trial. J Oral Rehabil. 2015;42(10):723-732.

42. Klein MM, Treister R, Raij T, et al. Transcranial magnetic stimulation of the brain: guidelines for pain treatment research. Pain. 2015;156(9): $1601-1614$

43. Lefaucheur JP, Hatem S, Nineb A, et al. Somatotopic organization of the analgesic effects of motor cortex rTMS in neuropathic pain. Neurology. 2006;67(11):1998-2004.

44. Jette F, Cote I, Meziane HB, Mercier C. Effect of single-session repetitive transcranial magnetic stimulation applied over the hand versus leg motor area on pain after spinal cord injury. Neurorehabil Neural Repair. 2013;27(7):636-643.

45. Short EB, Borckardt JJ, Anderson BS, et al. Ten sessions of adjunctive left prefrontal rTMS significantly reduces fibromyalgia pain: a randomized, controlled pilot study. Pain. 2011;152(11):2477-2484.

46. Lee SJ, Kim DY, Chun MH, Kim YG. The effect of repetitive transcranial magnetic stimulation on fibromyalgia: a randomized sham-controlled trial with 1-mo follow-up. Am J Phys Med Rehabil. 2012;91(12):1077-1085.

47. Legrain V, Iannetti GD, Plaghki L, Mouraux A. The pain matrix reloaded: a salience detection system for the body. Prog Neurobiol. 2011;93(1):111-124.
48. Peltz E, Seifert F, DeCol R, Dorfler A, Schwab S, Maihofner C. Functional connectivity of the human insular cortex during noxious and innocuous thermal stimulation. Neuroimage. 2011;54(2):1324-1335.

49. Lefaucheur JP, Antal A, Ahdab R, et al. The use of repetitive transcranial magnetic stimulation (rTMS) and transcranial direct current stimulation (tDCS) to relieve pain. Brain Stimulat. 2008;1(4):337-344.

50. Romero-Reyes M, Uyanik JM. Orofacial pain management: current perspectives. J Pain Res. 2014;7:99-115.

51. Picarelli H, Teixeira MJ, de Andrade DC, et al. Repetitive transcranial magnetic stimulation is efficacious as an add-on to pharmacological therapy in complex regional pain syndrome (CRPS) type I. J Pain. 2010;11(11):1203-1210.

52. Liebetanz D, Nitsche MA, Tergau F, Paulus W. Pharmacological approach to the mechanisms of transcranial DC-stimulation-induced after-effects of human motor cortex excitability. Brain. 2002;125(pt 10):2238-2247.

53. Nitsche MA, Fricke K, Henschke U, et al. Pharmacological modulation of cortical excitability shifts induced by transcranial direct current stimulation in humans. J Physiol. 2003;553(pt 1):293-301.

54. McLaren ME, Nissim NR, Woods AJ. The effects of medication use in transcranial direct current stimulation: a brief review. Brain Stimul. 2018;11(1):52-58.

55. Inghilleri M, Conte A, Frasca V, et al. Antiepileptic drugs and cortical excitability: a study with repetitive transcranial stimulation. Exp Brain Res. 2004;154(4):488-493.

56. Inghilleri M, Gilio F, Conte A, et al. Topiramate and cortical excitability in humans: a study with repetitive transcranial magnetic stimulation. Exp Brain Res. 2006;174(4):667-672.

57. Borckardt JJ, Reeves ST, Milliken C, et al. Prefrontal versus motor cortex transcranial direct current stimulation (tDCS) effects on post-surgical opioid use. Brain Stimulat. 2017;10(6):1096-1101.

58. Khedr EM, Sharkawy ESA, Attia AMA, Ibrahim Osman NM, Sayed ZM. Role of transcranial direct current stimulation on reduction of postsurgical opioid consumption and pain in total knee arthroplasty: double randomized clinical trial. Eur J Pain. 2017;21(8):1355-1365.

59. Coles AS, Kozak K, George TP. A review of brain stimulation methods to treat substance use disorders. Am J Addict. 2018;27(2):71-91.

60. Wiffen P, Collins S, McQuay H, Carroll D, Jadad A, Moore A. Anticonvulsant drugs for acute and chronic pain. Cochrane Database Syst Rev. 2005;(3):CD001133.

61. Harden RN, Weinland SR, Remble TA, et al. Medication quantification scale version III: update in medication classes and revised detriment weights by survey of American Pain Society Physicians. J Pain. 2005;6(6):364-371.

62. Song F, Parekh S, Hooper L, et al. Dissemination and publication of research findings: an updated review of related biases. Health Technol Assess. 2010;14(8):iii,ix-xi,1-193.

63. Davis NJ, Gold E, Pascual-Leone A, Bracewell RM. Challenges of proper placebo control for non-invasive brain stimulation in clinical and experimental applications. Eur J Neurosci. 2013;38(7):2973-2977.

64. Klasser GD, Manfredini D, Goulet JP, De Laat A. Oro-facial pain and temporomandibular disorders classification systems: a critical appraisal and future directions. J Oral Rehabil. 2018;45(3):258-268.

65. Lan L, Zhang X, Li X, Rong X, Peng Y. The efficacy of transcranial magnetic stimulation on migraine: a meta-analysis of randomized controlled trails. J Headache Pain. 2017;18(1):86.

66. Balasubramaniam R, Klasser GD, Delcanho R. Trigeminal autonomic cephalalgias: a review and implications for dentistry. JAm Dent Assoc. 2008;139(12):1616-1624. 
The Journal of Pain Research is an international, peer reviewed, open access, online journal that welcomes laboratory and clinical findings in the fields of pain research and the prevention and management of pain. Original research, reviews, symposium reports, hypothesis formation and commentaries are all considered for publication
The manuscript management system is completely online and includes a very quick and fair peer-review system, which is all easy to use. Visit http://www.dovepress.com/testimonials.php to read real quotes from published authors.

Submit your manuscript here: https://www.dovepress.com/journal-of-pain-research-journal 